\title{
Society of Neurological Surgeons boot camp courses: knowledge retention and relevance of hands-on learning after 6 months of postgraduate year 1 training
}

\author{
Clinical article
}

\author{
Nathan R. Selden, M.D., Ph.D., ${ }^{1}$ Valerie C. Anderson, Ph.D., M.C.R., ${ }^{1}$ \\ Shirley McCartney, Ph.D., ${ }^{1}$ Thomas C. Origitano, M.D., Ph.D., ${ }^{2}$ \\ Kim J. Burchiel, M.D., ${ }^{1}$ AND Nicholas M. Barbaro, M.D. ${ }^{3}$ \\ ${ }^{1}$ Department of Neurological Surgery, Oregon Health \& Science University, Portland, Oregon; ${ }^{2}$ Department \\ of Neurological Surgery, Loyola University Medical Center, Maywood, Illinois; and ${ }^{3}$ Department of \\ Neurological Surgery, University of California, San Francisco, California
}

Object. In July 2010, the Society of Neurological Surgeons (SNS) introduced regional courses to promote patient safety and teach fundamental skills and knowledge to all postgraduate Year 1 (PGY1) trainees entering Accreditation Council for Graduate Medical Education (ACGME)-accredited US neurosurgery residency programs. Data from these courses demonstrated significant didactic learning and high faculty and resident satisfaction with hands-on training. Here, the authors evaluated the durability of learning from and the relevance of participation in SNS PGY1 courses as measured midway through PGY1 training.

Methods. Resident participants were resurveyed 6 months after boot camp course attendance to assess knowledge retention and course effectiveness. Exposure to relevant hands-on experiences during PGY1 training and the subjective value of pre-residency simulated training in the courses were assessed.

Results. Ninety-four percent of all residents entering US PGY1 neurosurgical training participated in the 2010 SNS boot camp courses. One hundred sixty-four $(88 \%)$ of these resident participants responded to the survey. Six months after course completion, $99 \%$ of respondents believed the boot camp courses benefited beginning neurosurgery residents and imparted skills and knowledge that would improve patient care. The PGY1 residents' knowledge of information taught in the courses was retained 6 months after initial testing $(p<0.0001)$.

Conclusions. The learning and other benefits of participation in a national curriculum for residents entering PGY1 neurosurgical training were maintained 6 months after the courses, halfway through the initial training year. (http://thejns.org/doi/abs/10.3171/2013.3.JNS122114)

\section{KEY WORDS • boot camp • education • neurological surgery $\bullet$ residency $\bullet$ PGY1}

I N July 2010, ACGME-accredited neurosurgical training programs assumed direct responsibility for teaching fundamental skills and knowledge related to professionalism, supervision, communications, safety, and basic intensive care and operative procedures to PGY1 residents entering training. The SNS, comprising residency program directors, department chairs, and other educational leaders, created a uniform national curriculum for boot camp courses taught at 6 regional centers. ${ }^{15,16}$

Although measures of learning at these courses and the initial responses of resident and faculty participants were overwhelmingly positive, ${ }^{16}$ little is known about the durability of learning and the perceived relevance of

\footnotetext{
Abbreviations used in this paper: $\mathrm{ACGME}=$ Accreditation Council for Graduate Medical Education; EVD = external ventricular drain; ICP = intracranial pressure; PGY1 = postgraduate Year 1; $\mathrm{SNS}=$ Society of Neurological Surgeons; VP = ventriculoperitoneal.
}

hands-on training as measured later, after a period of exposure to the PGY1 training environment.

Therefore, after 6 months of PGY1 training, the PGY1 resident participants in all 6 regional courses from July 2010 were resurveyed regarding the SNS PGY1 boot camp course experience, the relevance of procedural skills training, and the retention of knowledge items taught during the courses. The value of hands-on participation exercises was evaluated in light of self-reported experience with similar procedures during PGY1 training.

\section{Methods}

The SNS PGY1 boot camp courses were designed, organized, and taught as previously described. ${ }^{16}$ The regional courses were held over 3 weekends following July $4,2010-2$ courses on each weekend. The courses began at 1 p.m. on a Friday to allow participants to arrive the 
same day. On Friday, the course consisted of nine 30-minute didactic lectures, followed by a course dinner for faculty and residents. On Saturday, the course included 3.5 hours each of procedural and craniotomy hands-on learning. All corporate funding and materials were provided via unrestricted educational grants to the 6 centers hosting the courses. ${ }^{16}$ No funds or materials beyond those directly required for the administration of the boot camp courses were disbursed.

Precourse, immediate postcourse, and 6-month postcourse surveys for resident participants were centrally designed and implemented using Survey Monkey (http:// www.surveymonkey.com) and distributed directly to individual residents using SNS resident email listings, with reminders as necessary. Resident postcourse surveys contained questions on overall aspects of the course and on specific curricular elements, including determination of how many times a resident performed a simulated procedure in the live clinical environment during the first 6 months after the course (none, 1, 2-3, 4-6, > 6), how confident residents were in completing the relevant procedure (confident, can always complete; competent, can usually complete; early learner, can sometimes complete; cannot complete; not applicable), whether the resident has experienced complications performing the procedure in the live clinical environment, and how much the courses enhanced the resident's ability to safely perform the procedure in the clinical environment (none; a little; a moderate amount; a lot; not applicable; see Appendix).

The surveys also repeated assessment of learning about 15 neurosurgical facts taught (11 facts) or not taught (4 facts) during the didactic portion of the courses. Finally, the surveys contained open-ended questions related to course improvement. Resident surveys were confidential, as mentioned at the course introduction and in the introductory text of each of the surveys themselves.

Statistical analyses were performed in Stata 12 (StataCorp LP). Associations between categorical variables were assessed using the Pearson chi-square analysis or Fisher exact test. Differences in the proportion of correct responses to items tested before and 6 months after the boot camp were compared using the McNemar test. The $p$ values were calculated at the 0.05 significance level in 2-tailed tests and are uncorrected for multiple comparisons.

\section{Results}

One hundred percent (186/186) of resident participants completed pre- and immediate postcourse surveys. One hundred sixty-four of these participants $(88 \%)$ also completed the 6-month postcourse survey. In the 6-month postcourse survey, $99 \%$ of respondents indicated that the course benefited beginning neurosurgery residents and that participation had improved patient care. Ninety-eight percent indicated that the course had met its learning objectives. These positive response rates were virtually identical to the immediate postcourse responses. ${ }^{16}$

The proportion of correct answers to knowledge questions taught during the courses increased from $72 \%$ before the courses to $83 \% 6$ months after the courses (p $<0.0001)$. The proportion of correct answers for items not taught during the courses increased from $54 \%$ before to $61 \% 6$ months after the courses ( $<<0.001)$. In both cases these are highly significant increases in the proportion correct from before to 6 months after the courses, although the absolute increases and the proportion correct are both higher for the taught than the not-taught items $(\mathrm{p}<0.04)$.

Among residents who had completed 2 or more lumbar drain procedures during PGY1 training, those who are confident and can always complete the procedure were significantly more likely to state that their course experience with simulated lumbar drain placement improved their ability "a moderate amount or a lot" (73\%), as compared with those who can only sometimes complete the procedure in practice $(33 \% ; \mathrm{p}<0.05)$. No such relationship existed for EVD placement, ICP monitor placement, VP shunt tap, or cervical traction.

Among the 148 residents who completed 2 or more EVD placement procedures during the first 6 months of PGY1 training, 33 (22\%) reported experiencing a complication. Those who experienced a complication were more likely to state that their boot camp experience with simulated EVD placement improved their ability to perform the procedure during PGY1 training "a moderate amount or a lot" (70\%) or "none" (12\%), as compared with those who did not experience a complication ("a moderate amount or a lot" $63 \%$, or "none" $3 \%$ ). Although the distribution of responses was significantly different between those who did and those who did not experience an EVD complication, individual comparisons were not $(\mathrm{p}<0.05)$. By contrast, only $6(7 \%)$ of 86 residents who reported 2 or more ICP monitor placement procedures also reported a complication, a proportion too small to analyze further. Similarly, only $5(5 \%)$ of 95 of those who reported 2 or more lumbar drain placements and $6(6 \%)$ of 99 who reported 2 or more VP shunt taps actually experienced a complication. Fewer PGY1 residents participated in cervical traction procedures during their first 6 months of training. Of the 59 residents who reported at least 1 traction procedure, only $1(2 \%)$ also reported experiencing a complication.

Four residents (2\%) identified unintended consequences of the courses: fatigue, "getting into trouble" for using a technique different from that of their home institution, requiring coverage for course attendance, and returning during the middle of a call shift after course attendance.

Procedures performed more frequently during PGY1 training (measured as the percentage of residents performing 2 or more procedures in the first 6 months of PGY1 training) were more highly valued; that is, residents benefited "a moderate amount" or "a lot" from simulated training during the boot camp courses (Fig. 1).

Extensive written responses to open-ended survey questions were also generated by resident participants. Among the most common comments were praise for the boot camp concept and implementation, requests for access to a detailed written curriculum for prestudy and review, access to lecture projection sets for review, and creation of a subsequent course for PGY2 or PGY3 residents to emphasize the transition to operative, research, and leadership skills.

Partly in response to these comments as well as feedback from faculty surveys, ${ }^{16}$ the SNS Subcommittee on 


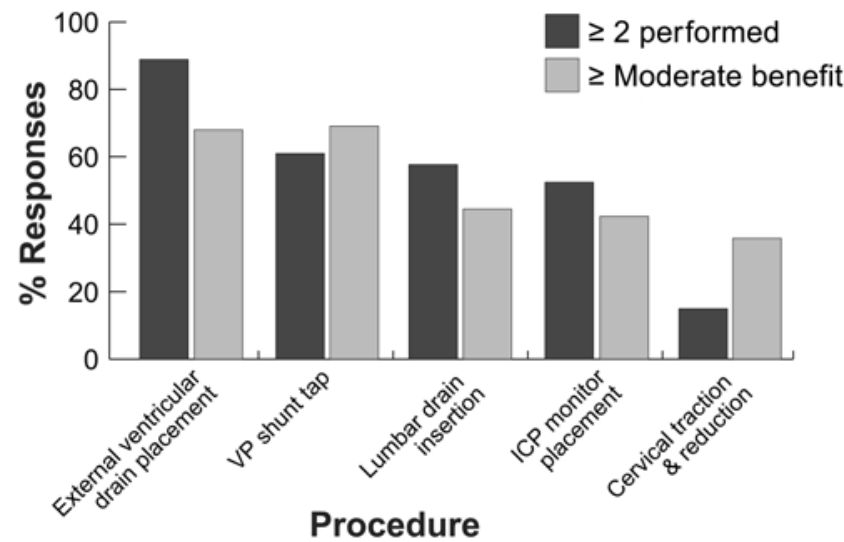

FIG. 1. Graph showing the benefit of simulated procedural learning, assessed after 6 months of PGY1 training. The percentage of respondents who performed the listed procedure 2 or more times is indicated by the dark gray bars. The percentage of residents who identified simulated skills training in that procedure during the PGY1 boot camp courses as enhancing their ability to perform the procedure "a lot" or "a moderate amount" during the first half of their PGY1 is indicated by the light gray bars.

Boot Camp Courses distributed printed and electronic copies of a course handbook beginning with the 2011 courses, including detailed procedural and operative skills instructions and goals, copies of curricular material, and other background information. Narrated digital course lectures were also reproduced online at the SNS boot camp website (http://www.societyns.org/BootCamp/ BootCampCourses.asp).

During the 2nd and 3rd years (2011 and 2012) of the national boot camp courses sponsored by the SNS, central administration of course finances, material, and travel reimbursement in collaboration with the Congress of Neurological Surgeons helped to enable $100 \%$ attendance by 201 (in 2011) and 206 (in 2012) entering US PGY1 residents in all 101 ACGME-accredited programs.

\section{Discussion}

The inaugural year of the SNS boot camp courses for entering neurosurgery trainees saw attendance by nearly all PGY1 residents at ACGME-accredited programs and evolved to $100 \%$ attendance in the courses' 2 nd year.

One hundred percent of resident participants completed surveys both before and immediately after course participation. Eighty-eight percent of these residents also completed a 6-month postcourse survey. Nearly universally positive course reviews by residents immediately after participation were sustained after 6 months of PGY1 clinical experience.

Knowledge of items explicitly taught during the didactic portion of the courses was retained or slightly enhanced 6 months later (78\% during immediate postcourse testing and 83\% during 6-month postcourse testing). Residents subjectively valued hands-on training most for bedside procedures they performed most frequently during the first 6 months of PGY1 training (Fig. 1).

For EVD placement, ICP monitor placement, VP shunt tap, and cervical traction, there was no relation- ship between the perceived value of hands-on training for individual procedures and a resident's later ability to effectively complete the procedure in the live clinical environment. Residents who highly valued lumbar drain placement training, however, were more likely to self-report competence in placing lumbar drains during PGY1 clinical practice.

Self-reported experience of a complication during the performance of bedside procedures was rare for most procedures during the first 6 months of the PGY1, with the exception of EVD placement (22\%). This finding is consistent with reported complication rates for these procedures. ${ }^{2,3,6,9-13,23}$ Compared with residents who had not experienced a complication during EVD placement, those who did report a complication tended to either highly value or not value their simulated EVD placement experience during the boot camp courses, presumably depending on whether they emphasized the occurrence of the complication or their ability to understand and manage it. Because of low complication rates, no such relationship could be evaluated for ICP monitor placement, lumbar drain placement, VP shunt tap, or cervical traction.

The SNS Subcommittee on Boot Camp Courses has pursued a model of iterative course evaluation and improvement. The principal current undertaking of the committee is to develop and introduce validated skills assessments for several of the hands-on portions of the course, which are typically lacking in the evaluation of surgical training methodologies. ${ }^{5,19}$ Although difficult in practice, the ultimate goal is to demonstrate a relationship between introductory simulated training and safe performance of procedures and operative skills in the live clinical environment. ${ }^{8,9,14,17,18,20,21}$

The ACGME now recognizes that structured education equivalent to participation in the SNS PGY1 boot camp courses is a required activity before PGY1 residents can be indirectly supervised for procedures and patient management competencies. ${ }^{1}$ Many residency training programs, including one directed by the senior author, use simulated experience at the boot camp courses as a prerequisite for further training, observation, and safety validation in the live clinical environment at the home program, before approving performance of these procedures with indirect supervision.

Some of the most important duties early in residency training involve responses to sudden changes in clinical status, urgent decision making, and team and interdisciplinary communication. ${ }^{4,7,22}$ The PGY1 boot camp courses now include a simulated clinical crisis, that is, neurological deterioration in the emergency department due to an expanding epidural hematoma. Trained educators carry out the scenario using digitally controlled manikins that simulate vital sign and physical examination changes, critical care monitors, and axial imaging displays.

Curricular material for the courses is available for residents and faculty in various online forums. A course handbook containing background material, as well as detailed curricular goals and technical steps for each handson and operative skills station, is distributed to all course participants either electronically in advance (http://www. societyns.org/BootCamp/BootCampCourses.asp) or in print at the courses. Didactic material is also available 
online in the form of centrally reviewed and standardized digital presentations with faculty narration (https://itunes. apple.com/us/itunes-u/aans-sns-neurosurgical-online/ id439288626). These resources assist faculty in preparing to teach the courses and residents in both preparatory and consolidative review.

\section{Conclusions}

The boot camp courses provide systematic, specialty-wide training in core elements of knowledge, procedural skills, professionalism, supervision, and communications for residents entering neurological surgery. The benefits of boot camp participation appear to persist at least through the first half of the PGY1 training year. The courses are most valued for and may have the greatest impact on those procedures most commonly performed during PGY1 training. Iterative course evaluations and improvements have strengthened the curriculum and served as a template for further curricular and practical course development by the SNS.

\section{Disclosure}

Dr. Selden serves as chair of the SNS Subcommittee on Boot Camp Courses and as secretary of the CNS. Dr. Barbaro serves as chair of the SNS Committee on Resident Education. Dr. Burchiel serves as secretary of the SNS. Course directors and faculty received no honoraria or other incentives for participation. The authors have no personal financial or institutional interest in any of the drugs, materials, or devices described in this article. The SNS PGY1 Boot Camp courses were entirely funded by individual unrestricted educational grants from Stryker to each of the 6 regional center host institutions, plus in-kind material donations and equipment loans from Stryker, Medtronic, Integra Neurosciences, Carl Zeiss, Ethicon, and Teleflex. No funds or materials beyond those required directly for the administration of the boot camp courses were disbursed.

Author contributions to the study and manuscript preparation include the following. Conception and design: Selden, McCartney, Origitano, Burchiel, Barbaro. Acquisition of data: Selden, McCartney. Analysis and interpretation of data: Selden, Anderson. Drafting the article: Selden. Critically revising the article: all authors. Reviewed submitted version of manuscript: all authors. Approved the final version of the manuscript on behalf of all authors: Selden. Statistical analysis: Anderson.

\section{Acknowledgments}

The authors acknowledge the SNS Boot Camp faculty, who have given tirelessly of their time and expertise to advance our specialty through education; the SNS Executive Council, who have supported this endeavor throughout; the corporate educational sponsors of the courses (Stryker, Medtronic, Integra Neurosciences, Ethicon, Teleflex, and Carl Zeiss); the administrative organizers at the 6 course centers; the course directors (Drs. Nicholas Barbaro, Daniel Barrow, Bruce Ehni, M. Sean Grady, Costas Hadjipanayis, Carl Heilman, Thomas Origitano, A. John Popp, Raymond Sawaya, Nathan Selden, James Schuster, and Julian $\mathrm{Wu}$ ); and the courses' administrative sponsor, the CNS (in particular, Ms. Lisa O'Brien and Ms. Regina Shupak).

Finally, the authors dedicate this publication to our late colleague, one of the early organizers of the boot camp courses, founding co-director of the Chicago SNS PGY1 Boot Camp Course, and founding member of the Boot Camp Courses Steering Committee, Dr. Christopher Getch.

\section{References}

1. Accreditation Council for Graduate Medical Education: Frequently Asked Questions: Neurological Surgery. (http:// www.acgme.org/acgmeweb/Portals/0/PDFs/FAQ/160_ Neurological_Surgery_FAQs.pdf) [Accessed March 14, 2013]

2. Anderson RC, Kan P, Klimo P, Brockmeyer DL, Walker ML, Kestle JR: Complications of intracranial pressure monitoring in children with head trauma. J Neurosurg 101 (1 Suppl): 53-58, 2004

3. Coplin WM, Avellino AM, Kim DK, Winn HR, Grady MS: Bacterial meningitis associated with lumbar drains: a retrospective cohort study. J Neurol Neurosurg Psychiatry 67: 468-473, 1999

4. Daniels K, Lipman S, Harney K, Arafeh J, Druzin M: Use of simulation based team training for obstetric crises in resident education. Simul Healthc 3:154-160, 2008

5. Hogle NJ, Chang L, Strong VE, Welcome AO, Sinaan M, Bailey $\mathrm{R}$, et al: Validation of laparoscopic surgical skills training outside the operating room: a long road. Surg Endosc 23: 1476-1482, 2009

6. Kitchen WJ, Singh N, Hulme S, Galea J, Patel HC, King AT: External ventricular drain infection: improved technique can reduce infection rates. Br J Neurosurg 25:632-635, 2011

7. Knudson MM, Khaw L, Bullard MK, Dicker R, Cohen MJ, Staudenmayer K, et al: Trauma training in simulation: translating skills from SIM time to real time. J Trauma 64:255264, 2008

8. Larsen CR, Soerensen JL, Grantcharov TP, Dalsgaard T, Schouenborg L, Ottosen C, et al: Effect of virtual reality training on laparoscopic surgery: randomised controlled trial. BMJ 338:b1802, 2009 (Erratum in BMJ 338:b2074, 2009)

9. Mishra S, Kurien A, Patel R, Patil P, Ganpule A, Muthu V, et al: Validation of virtual reality simulation for percutaneous renal access training. J Endourol 24:635-640, 2010

10. Morton R, Lucas TH II, Ko A, Browd SR, Ellenbogen RG, Chesnut RM: Intracerebral abscess associated with the Camino intracranial pressure monitor: case report and review of the literature. Neurosurgery 71:E193-E198, 2012

11. Münch E, Weigel R, Schmiedek P, Schürer L: The Camino intracranial pressure device in clinical practice: reliability, handling characteristics and complications. Acta Neurochir (Wien) 140:1113-1120, 1998

12. Ngo QN, Ranger A, Singh RN, Kornecki A, Seabrook JA, Fraser DD: External ventricular drains in pediatric patients. Pediatr Crit Care Med 10:346-351, 2009

13. Noetzel MJ, Baker RP: Shunt fluid examination: risks and benefits in the evaluation of shunt malfunction and infection. J Neurosurg 61:328-332, 1984

14. Schout BM, Ananias HJ, Bemelmans BL, d'Ancona FC, Muijtjens AM, Dolmans VE, et al: Transfer of cysto-urethroscopy skills from a virtual-reality simulator to the operating room: a randomized controlled trial. BJU Int 106:226-231, 2010

15. Selden NR, Barbaro N, Origitano TC, Burchiel KJ: Fundamental skills for entering neurosurgery residents: report of a Pacific region "boot camp" pilot course, 2009. Neurosurgery 68:759-764, 2011

16. Selden NR, Origitano TC, Burchiel KJ, Getch CC, Anderson VC, McCartney S, et al: A national fundamentals curriculum for neurosurgery PGY1 residents: the 2010 Society of Neurological Surgeons boot camp courses. Neurosurgery 70: 971-981, 2012

17. Sroka G, Feldman LS, Vassiliou MC, Kaneva PA, Fayez R, Fried GM: Fundamentals of laparoscopic surgery simulator training to proficiency improves laparoscopic performance in the operating room-a randomized controlled trial. Am J Surg 199:115-120, 2010

18. Stefanidis D, Acker C, Heniford BT: Proficiency-based laparoscopic simulator training leads to improved operating room skill that is resistant to decay. Surg Innov 15:69-73, 2008 
N. R. Selden et al.

19. van Hove PD, Tuijthof GJ, Verdaasdonk EG, Stassen LP, Dankelman J: Objective assessment of technical surgical skills. Br J Surg 97:972-987, 2010

20. Van Sickle KR, Ritter EM, Baghai M, Goldenberg AE, Huang IP, Gallagher AG, et al: Prospective, randomized, doubleblind trial of curriculum-based training for intracorporeal suturing and knot tying. J Am Coll Surg 207:560-568, 2008

21. Vankipuram M, Kahol K, McLaren A, Panchanathan S: A virtual reality simulator for orthopedic basic skills: a design and validation study. J Biomed Inform 43:661-668, 2010

22. Volk MS, Ward J, Irias N, Navedo A, Pollart J, Weinstock PH: Using medical simulation to teach crisis resource management and decision-making skills to otolaryngology housestaff. Otolaryngol Head Neck Surg 145:35-42, 2011

23. Wiesmann M, Mayer TE: Intracranial bleeding rates associated with two methods of external ventricular drainage. J Clin Neurosci 8:126-128, 2001

\section{Appendix: 2010 Post-SNS boot camp questions}

1. Identification (confidential, for course design and improvement only-data will not be shared with your individual residency program).

Answer Options

Name:

Residency Program:

State:

Email Address:

2. Impairment of third cranial nerve function results in which of the following clinical findings?

Answer Options

Anosmia

Impaired vision

Facial weakness

Pupillary dilation

Absent shoulder shrug

3. Triceps weakness most often results from injury to which nerve root?

Answer Options

C-4

C-5

C-6

C-7

C-8

4. Cranial epidural hemorrhage most often originates from what vessel?

Answer Options

Carotid artery

Middle cerebral artery

Middle meningeal artery

Vein of Galen

Transverse sinus

5. Stereotactic lesion making in what basal ganglia nucleus is used to treat some cases of Parkinson disease?

Answer Options

Caudate nucleus

Nucleus accumbens

Nucleus basalis of Meynert

Globus pallidus

Putamen

6. In an adult, the normal interval between the anterior arch of the $C-1$ vertebra and the dens $(C-2)$ is?
Answer Options

$<3 \mathrm{~mm}$

4-5 $\mathrm{mm}$

6-7 mm

$8-9 \mathrm{~mm}$

$>10 \mathrm{~mm}$

7. What is the upper limit of normal for intracranial pressure (ICP) in an adult?

Answer Options

$0 \mathrm{~mm} \mathrm{Hg}$

$5 \mathrm{~mm} \mathrm{Hg}$

$10 \mathrm{~mm} \mathrm{Hg}$

$20 \mathrm{~mm} \mathrm{Hg}$

$50 \mathrm{~mm} \mathrm{Hg}$

8. The upper limit of serum osmolarity when using mannitol for ICP control is?

Answer Options

$260 \mathrm{mOsm} / \mathrm{L}$

$280 \mathrm{mOsm} / \mathrm{L}$

$300 \mathrm{mOsm} / \mathrm{L}$

$320 \mathrm{mOsm} / \mathrm{L}$

$340 \mathrm{mOsm} / \mathrm{L}$

9. Loss of what neurotransmitter is most closely associated with memory loss?

Answer Options

Acetylcholine

Dopamine

Glutamate

Norepinephrine

Serotonin

10. The appropriate loading dose of Dilantin for an adult patient presenting in status epilepticus is?

Answer Options

$1-3 \mathrm{mg} / \mathrm{kg}$

$5-10 \mathrm{mg} / \mathrm{kg}$

$15-20 \mathrm{mg} / \mathrm{kg}$

$25-30 \mathrm{mg} / \mathrm{kg}$

$50-100 \mathrm{mg} / \mathrm{kg}$ lus?

11. Aqueductal stenosis results in what type of hydrocepha-

Answer Options

Communicating

External

Normal Pressure

Ex vacuo

Triventricular

12. The major arteries supplying the scalp run in what layer?

Answer Options

Skin

Connective tissue

Aponeurosis (Galea aponeurotica)

Loose areolar tissue

Pericranium

13. The most common tumor of the posterior fossa in children is?

Answer Options

Ependymoma

Astrocytoma

Medulloblastoma

Hemangioblastoma

Teratoma 
14. During the informed consent process for a surgical procedure, the surgeon must disclose all risks that:

Answer Options

Have occurred in his or her previous patients

Are listed on the American College of Surgeons website for the procedure

Have ever occurred in any patient undergoing this procedure

Would influence a reasonable person in the same circumstance

Have been reported in medical journals since 1948 (the era covered by Medline)

15. Resident duty-hour restrictions have raised concerns about reduced continuity of patient care. In response, hospitals should:

\section{Answer Options}

Enhance the training of inpatient nursing staff

Improve systems for patient hand offs at shift change

Counsel patients about less personalized care

Lengthen overall residency training

Increase liability insurance coverage

16. The global services period for major surgical (including neurosurgical) procedures is most commonly:
10 days
20 days
One month
60 days
90 days

Answer Options

17. Since the boot camp course, how many external ventricular drain placement procedures have you performed?

$$
\begin{aligned}
& \text { None } \\
& 1 \\
& 2-3 \\
& 4-6 \\
& >6
\end{aligned}
$$$$
\text { Answer Options }
$$

18. My experience with external ventricular drain since the boot camp course is:

Answer Options

Confident, can always complete

Competent, can usually complete

Early learner, can sometimes complete

Cannot complete

Not Applicable

19. I have had complications associated with doing an external ventricular drain procedure:

Answer Options

Yes

No

Not Applicable

20. I feel the boot camp course enhanced my ability to safely perform an external ventricular drain procedure in the clinical setting:

Answer Options

None

A little

A moderate amount

A lot

Not Applicable

21. Since the boot camp course, how many ICP monitor (fiberoptic, "bolt") placement procedures have you performed?

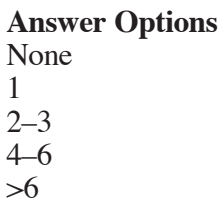

22. My experience with ICP monitor (fiberoptic, "bolt") placement procedures since the boot camp course is:

Answer Options

Confident, can always complete

Competent, can usually complete

Early learner, can sometimes complete

Cannot complete

Not Applicable

23. I have had complications associated with doing ICP monitor (fiberoptic, “bolt") placement procedures:

Answer Options

Yes

No

Not Applicable

24. I feel the boot camp course enhanced my ability to safely perform an ICP monitor (fiberoptic, "bolt") placement procedure in the clinical setting:

Answer Options

None

A little

A moderate amount

A lot

Not Applicable

25. Since the boot camp course, how many lumbar drain insertion procedures have you performed?

Answer Options

None

1

2-3

$4-6$

$>6$

26. My experience with lumbar drain insertion procedures since the boot camp course is:

Answer Options

Confident, can always complete

Competent, can usually complete

Early learner, can sometimes complete

Cannot complete

Not Applicable

27. I have had complications associated with doing lumbar drain insertion procedures:

Answer Options

Yes

No

Not Applicable

28. I feel the boot camp course enhanced my ability to safely perform lumbar drain insertion procedures in the clinical setting:

Answer Options

None

A little

A moderate amount

A lot

Not Applicable 
N. R. Selden et al.

29. Since the boot camp course, how many VP shunt tap procedures have you performed?

Answer Options

None

1

2-3

4-6

$>6$

30. My experience with VP shunt tap procedures since the boot camp course is:

Answer Options

Confident, can always complete

Competent, can usually complete

Early learner, can sometimes complete

Cannot complete

Not Applicable

31. I have had complications associated with doing VP shunt tap procedures:

Answer Options

Yes

No

Not Applicable

32. I feel the boot camp course enhanced my ability to safely perform VP shunt tap procedures in the clinical setting:

Answer Options

None

A little

A moderate amount

A lot

Not Applicable

33. Since the boot camp course, how many cervical traction/ reduction procedures have you performed?

Answer Options

None

1

$2-3$

$4-6$

$>6$

34. My experience with cervical traction/reduction procedures since the boot camp course is:

\section{Answer Options}

Confident, can always complete

Competent, can usually complete

Early learner, can sometimes complete

Cannot complete

Not Applicable

35. I have had complications associated with doing cervical traction/reduction procedures:

Answer Options

Yes

No

Not Applicable

36. I feel the boot camp course enhanced my ability to safely perform cervical traction/reduction procedures in the clinical setting:
Answer Options

None

A little

A moderate amount

A lot

Not Applicable

37. Do you feel the boot camp course benefits beginning neurosurgery residents?

Answer Options

Yes

No

Please explain why.

38. What did you think was most beneficial about the course?

39. What did you think was least beneficial about the course?

40. When should the course be offered (currently July)?

Answer Options

A = July

$\mathrm{B}=$ August

$\mathrm{C}=$ Autumn

$\mathrm{D}=$ Winter

$\mathrm{E}=$ Spring tives?

41. Did the course adequately fulfill its purpose and objec-

Answer Options

Yes

No

42. Did the knowledge and skills learned help you to improve patient care?

Answer Options

Yes

No

43. Did any aspect of the course or participation have unintended negative consequences?

Answer Options

Yes

No

If so, what were the negative consequences?

44. Did your home program teach an alternate method for any of the above procedures?

Answer Options

Yes

No

If so, please list the procedure(s) and state the differences.

45. Other comments/feedback?

Manuscript submitted November 6, 2012.

Accepted March 12, 2013.

Please include this information when citing this paper: published online April 16, 2013; DOI: 10.3171/2013.3.JNS122114.

Address correspondence to: Nathan R. Selden, M.D., Ph.D., Department of Neurological Surgery, Oregon Health \& Science University, 3303 SW Bond Avenue, CH8N, Portland, Oregon 97239. email: seldenn@ohsu.edu. 